\title{
Hábitos del usuario y tipo de red social como predictores de consumo y difusión de noticias
}

\section{User habits and social media type as predictors of news consumption and sharing}

\author{
Jesús Díaz-Campo; Francisco Segado-Boj; Erika Fernández-Gómez
}

Note: This article can be read in English on:

https://revista.profesionaldelainformacion.com/index.php/EPI/article/view/86436

Cómo citar este artículo:

Díaz-Campo, Jesús; Segado-Boj, Francisco; Fernández-Gómez, Erika (2021). “User habits and social media type as predictors of news consumption and sharing". Profesional de la información, v. 30, n. 4, e300417.

https://doi.org/10.3145/epi.2021.jul.17

Artículo recibido el 14-03-2021

Aceptación definitiva: 27-05-2021

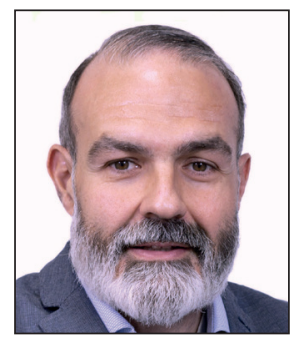

Jesús Díaz-Campo

https://orcid.org/0000-0001-5014-8749

Universidad Internacional de La Rioja Facultad de Empresa y Comunicación Av. de la Paz, 137

26006 Logroño, España

jesus.diaz@unir.net

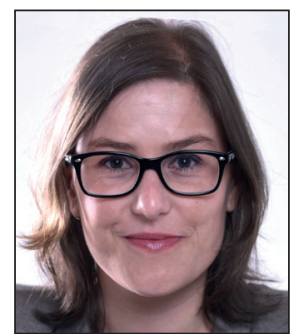

\author{
Erika Fernández-Gómez \\ https://orcid.org/0000-0002-7088-1814 \\ Universidad Internacional de La Rioja \\ Facultad de Empresa y Comunicación \\ Av. de la Paz, 137 \\ 26006 Logroño, España \\ erika.fernandez@unir.net
}

\section{Resumen}

Se analizan los patrones de comportamiento que siguen los usuarios a la hora de consumir y difundir información en redes sociales, prestando especial atención a cómo influye el tipo de exposición y el tipo de red social. Se utilizó el método de muestreo de experiencias, de uso habitual para analizar la actividad en las redes sociales, que consiste en que un grupo de participantes voluntarios debe contestar, en distintos momentos temporales, a un conjunto de preguntas sobre alguna actividad que hayan realizado o alguna experiencia que hayan tenido. La muestra estuvo formada por 279 sujetos. Los resultados muestran que las noticias que requieren un mayor esfuerzo suelen compartirse en redes cerradas, mientras que en las redes abiertas se tiende a compartir información más superficial. No existen diferencias significativas entre el grado de lectura de la noticia y el tipo de red social donde se comparte; el nivel de consumo no influye en el grado de lectura de las que se comparten. Se ha comprobado que el nivel de disfrute de las noticias influye en su grado de lectura. Respecto a las diferencias entre redes, destaca el hecho de que en Facebook la frecuencia de uso influye en las dinámicas de consumo y difusión, y cómo las noticias compartidas tienen más afecto y utilidad. Por el contrario, en el caso de Twitter existe una mayor preferencia hacia las informaciones consideradas blandas.

\section{Palabras clave}

Redes sociales; Medios sociales; Consumo de noticias; Difusión de noticias; Facebook; Twitter; WhatsApp; Noticias; Periodismo digital; Viralidad; Exposición incidental.

\section{Abstract}

User behavior patterns when consuming and sharing information on social networks are analyzed, paying special attention to the effects of the type of presentation and the type of social network. As is common when analyzing activity 
on social networks, the experience sampling method was used, in which a group of volunteer participants are asked, at different times, a set of questions about their recent activity or experience. The sample consisted of 279 subjects. The results show that news that requires a greater effort is usually shared through closed networks, while in open networks there is a tendency to share more superficial information. No significant differences are found between the degree of reading of the news and the type of social network where it is shared. Moreover, the level of consumption does not influence the degree of reading of the shared material. The level of enjoyment of the news is found to influence its degree of reading. The differences between networks highlight that, on Facebook, the frequency of use influences the dynamics of consumption and sharing, with shared news being more impactful and useful. In contrast, in the case of Twitter, there is a greater preference for so-called soft information.

\section{Keywords}

Social networks; Social media; News consumption; News dissemination; Facebook; Twitter; WhatsApp; News; Digital journalism; Virality; Incidental exposure.

\section{Financiación}

Esta investigación es un resultado del proyecto Consumo de noticias en medios sociales. Análisis de factores en la selección y difusión de contenidos mediáticos, referencia CSO2017-86312-R financiado por el Ministerio de Economía, Industria y Competitividad (Mineco), la Agencia Estatal de Investigación (AEI) y el Fondo Europeo de Desarrollo Regional (Feder), dentro de la convocatoria 2017 de ayudas a proyectos de I+D+I correspondientes al Programa Estatal de Investigación, Desarrollo e Innovación Orientada a los Retos de la Sociedad en el marco del Plan Estatal de Investigación Científica y Técnica y de Innovación 2013-2016.

http://newssharing.es

\section{Introducción}

El ecosistema de medios de comunicación vive en los últimos tiempos una profunda transformación que se traduce en una serie de cambios en las dinámicas de distribución y consumo de noticias por parte de los ciudadanos. Se trata de modificaciones que afectan tanto a las fuentes de las que se extrae esa información como a la naturaleza de las noticias que se consumen (Jansson; Lindell, 2015). De este modo, las plataformas y canales se multiplican, los contenidos circulan de forma mucho más imprevisible, generando una sobreabundancia informativa, y la línea que define lo que constituye una fuente relevante y de calidad resulta cada vez más difusa (McNeill, 2018).

Uno de los elementos que desempeña un papel más importante en estos procesos son las tecnologías de la información y la comunicación y, en especial, internet y las redes sociales, que se han convertido en un relevante canal de distribución de noticias (Bright, 2016; Dafonte-Gómez, 2018). Así, las últimas ediciones del Digital news reports que elabora el Reuters Institute (Newman et al., 2018; 2019; 2020) muestran una doble tendencia:

- descenso en el consumo de noticias a través de los medios tradicionales;

- aumento en el uso de las redes sociales como fuente informativa.

Al mismo tiempo, y como consecuencia de ello, se produce una transformación radical del modelo de distribución de las noticias, pasando del clásico paradigma unidireccional, en el que los medios de comunicación ejercían el control, a un nuevo paradigma multidireccional en el que los propios usuarios controlan la difusión (Noguera-Vivo, 2018), ya que son ellos los que deciden qué contenidos concretos comparten con otros usuarios con los que están en contacto en las redes sociales. Por tanto, la transmisión es mucho más compleja, y no se basa únicamente en una línea que va del emisor al receptor, sino que se articula en una red en la que los usuarios constituyen nodos que redistribuyen los contenidos mediáticos (Carlson, 2016; Guallar et al., 2016; Klinger; Svensson, 2016). Así, los usuarios con mejor disposición a utilizar las redes para buscar contenidos noticiosos y difundirlos posteriormente en ellas suelen ser personas con un alto consumo de noticias, que utilizan una variedad de fuentes y que suelen dedicar bastante tiempo a leer las noticias (Kümpel; Karnowski; Keyling, 2015).

Por tanto, factores como la participación del usuario, la conectividad permanente, la multipantalla o los dispositivos móviles son cada vez más determinantes en el consumo de noticias (Picone; Courtois; Paulussen, 2015; Peters, 2015). Es decir, se trata de un ejercicio que, por una parte, resulta cada vez más individual, ya que cada usuario dispone de su propio dispositivo digital, o incluso más de uno en algunos casos. Pero, por otro lado, y en cierto modo paradójicamente, el ejercicio adquiere tintes cada vez más sociales (Papacharissi, 2015), gracias a esa interconexión que se produce en las redes y plataformas.

De hecho, otro de los factores que influye es que el contenido se difunda a través de una red social abierta (tipo Facebook o Twitter) o cerrada (tipo WhatsApp) (Salaverría et al., 2020). Profundizando en esa distinción, Kim e Ihm (2019) diferencian entre redes sociales abiertas y asimétricas (RAA) y redes sociales cerradas y simétricas (RCS). En el primer caso, cuando un usuario publica un mensaje, todos sus contactos pueden verlo, de forma que no se distingue entre unos usuarios y otros (Yang, 2016). Esto provoca, entre otras cosas, que los usuarios practiquen la autocensura, esto es, que releguen aquellas noticias que consideren como potencialmente polémicas (Kim; Ihm, 2019). 
Mientras, en las RCS es el usuario el que toma la iniciativa al crearlas y controlar quién se puede unir a ellas. Por tanto, no están abiertas al público en general. Así ocurre con las aplicaciones de mensajería móvil o determinadas utilidades de las RAA, como los grupos privados. En este caso, la difusión de noticias sigue un patrón mucho más selectivo, ya que los mensajes van dirigidos a un grupo más reducido y específico de seguidores (Goncalves; Kostakos; Venkatanathan, 2013; Karapanos; Teixeira; Gouveia, 2016). En definitiva, la naturaleza de la red de la que forma parte el usuario influye en el tipo de contenido que se comparte (Kümpel; Karnowski; Keyling, 2015).

Las emociones también influyen a la hora de tomar esa decisión, de forma que los usuarios son más propensos a compartir los contenidos que generan en ellos un afecto más positivo (Bakshy et al., 2012) y, a su vez, estos contenidos generan un mayor interés en los usuarios que los reciben (Berger, 2011).

De este modo, es bastante común que los usuarios utilicen internet buscando, por ejemplo, contacto social o entretenimiento, pero acaben accediendo a las noticias, aunque no las estuvieran buscando de forma directa. Se trata de una tendencia en la que tiene mucho que ver la ubicuidad de las noticias en internet y que se traduce en que las noticias están ahí y "me encontrarán a mí" (Gil de Zúñiga; Weeks; Ardèvol-Abreu, 2017), una percepción cada vez más generalizada (Toff; Nielsen, 2018; Bergström; Jervelycke; Belfrage, 2018, Segado-Boj et al., 2020).

En definitiva, el ejercicio que realiza el usuario cuando se dispone a consultar un contenido noticioso en cualquier plataforma o medio digital puede seguir diferentes estrategias, algunas directas o intencionadas, y otras involuntarias e indirectas. En concreto, estas estrategias son fundamentalmente tres (Antunovic; Parsons; Cooke, 2018; Boczkowski; Mitchelstein; Matas, 2018; Gunter 2015; Molyneux, 2018; Schrøder, 2015):

- monitoreo o vigilancia rutinaria de las redes y plataformas: consiste en una especie de chequeo o comprobación constante, a través de los dispositivos móviles, de las noticias a las que el usuario tiene acceso (Antunovic; Parsons; Cooke, 2018);

- exposición incidental a las noticias: ha sido definida como la forma en que

"las personas encuentran información sobre asuntos actuales cuando no la habían estado buscando activamente" (Tewksbury; Weaver; Maddex, 2001, p. 534).

- consumo directo: es el que se realiza cuando los usuarios tienen especial interés en buscar información más detallada sobre alguna cuestión o contenido concreto (Antunovic; Parsons; Cooke, 2018).

La rutina de monitoreo alude a los usuarios que incluyen entre sus hábitos diarios el de intentar estar informados de lo que ocurre en el mundo, para lo cual consultan de manera regular una serie de fuentes informativas (Antunovic; Parsons; Cooke, 2018). Este proceso, que no resulta nuevo en cuanto a su naturaleza, sí que ha cambiado en lo que respecta a los soportes y fuentes informativas consultados. Lo que hace unas décadas se limitaba a la prensa escrita, la radio o la televisión, ahora incluye diversas modalidades, empezando por la visita habitual a la web del medio de comunicación, y siguiendo por aplicaciones, newsletters o servicios de alertas, que envían al usuario de manera automatizada resúmenes de noticias con lo más destacado que se publica en determinadas fuentes o que hace referencia a temas específicos (Yuan, 2011). De este modo el monitoreo de la actualidad asume una mayor cantidad de vertientes y modalidades

La estrategia más estudiada de las tres por la bibliografía académica es, con diferencia, la exposición incidental, que se introdujo ya a finales del siglo 20, en un contexto mediático caracterizado por el protagonismo de los medios impresos y audiovisuales (Erdelez, 1995). La aparición de internet y las redes sociales ha aumentado el interés investigador y los trabajos científicos en este sentido (Feezell, 2018; Fletcher; Nielsen, 2018; Kümpel, 2019).

A lo largo de los años, la percepción de la exposición incidental ha evolucionado, pasando por la capacidad de internet para poner al alcance del usuario una cantidad de contenidos informativos mucho mayor y más diversa de la que inicialmente buscaba (Tewksbury; Weaver; Maddex, 2001), a intentar localizar los factores que influyen más en el proceso, como las características del entorno mediático o las predisposiciones personales (Lee, 2009), y a centrarse más en la percepción, por parte del individuo, de que puede sentirse informado a pesar de no haber realizado un ejercicio activo de búsqueda de las noticias (Hermida, 2016; Hermida et al., 2012; Toff; Nielsen, 2018).

Las distintas investigaciones llevadas a cabo también han concluido que dos de los aspectos que correlacionan positivamente con la exposición incidental son la mayor o menor heterogeneidad de la red de contactos y el hecho de que los lazos débiles prevalezcan en esa red (Lee; Kim, 2017).

Por su parte, Fletcher y Nielsen (2018) concluyeron, en un trabajo en el que los usuarios eran de Estados Unidos, Reino Unido, Australia e Italia, que los individuos que se exponen a las noticias de forma incidental suelen acudir a un mayor número de fuentes digitales y que el efecto de este tipo de exposición es mayor en las personas más jóvenes y con menor interés previo en las noticias. 
Park y Kaye (2020), en un estudio referido a personas adultas de Corea del Sur, concluyeron que la exposición incidental refuerza la percepción de que las noticias me encuentran a mí y que, a su vez, esta percepción es un elemento que influye en la relación entre la exposición incidental y el consumo de noticias.

Asimismo, distintos autores han abordado los aspectos positivos de la exposición incidental, sobre todo en el ámbito de la opinión pública. Entre ellos cabe referirse a la posibilidad de conocer más en profundidad los asuntos públicos, aumentar el compromiso cívico de los individuos o contar con un mayor y más fácil acceso a fuentes de noticias más diversas (Bode, 2016; Feezell, 2018; Fletcher; Nielsen, 2018; Valeriani; Vaccari, 2016).

Por su parte, Thorson (2020) introduce una nueva perspectiva en el estudio del concepto y aboga por prestar mayor atención a la capacidad de las plataformas y los algoritmos para configurar la mayor o menor probabilidad de estar expuestos a las noticias a través de los medios digitales. En esta línea, insiste en la idea de la ubicuidad del contenido noticioso en las redes sociales y resalta que la exposición incidental no siempre implica exposición accidental, como en ocasiones se ha señalado en la bibliografía sobre esta cuestión (Prior, 2007; Valeriani; Vaccari, 2016). Esta autora sintetiza esta idea en el concepto de "atracción hacia las noticias".

Por último, el consumo directo supone una evolución con respecto al proceso de la rutina de monitoreo. Se produce cuando sucede un hecho que despierta el interés especial por parte del usuario, de modo que va más allá de su rutina de consultar determinadas fuentes o temas, y dedica más tiempo a buscar de manera concreta todo lo que tenga que ver con ese evento (Rubin; Perse, 1987).

Al igual que sucedía con la rutina de monitoreo, con el paso del tiempo la naturaleza de las fuentes a las que recurre el usuario también ha cambiado de manera sensible. Así, en la prensa escrita esto se traduciría en leer más allá del titular o la entradilla de la noticia. En el contexto digital, en pinchar en los enlaces a las noticias o incluso en informaciones relacionadas. Por último, cabe destacar que, si esta búsqueda se prolonga en el tiempo y se convierte en algo habitual, este proceso puede derivar y convertirse en la de rutina de monitoreo (Antunovic; Parsons; Cooke, 2018).

\section{Objetivos}

El objetivo general de esta investigación es analizar los patrones de comportamiento de los usuarios a la hora de consumir y difundir información en redes sociales, prestando especial atención a cómo influye

- el tipo de exposición: incidental, vigilancia rutinaria o consumo directo;

- el tipo de red social: abierta o cerrada.

A partir de ese objetivo se plantean las siguientes hipótesis:

H1. El tipo de exposición (incidental, rutinaria o directa) se relaciona con el grado de lectura de la noticia (en profundidad, superficialmente, o sólo el titular), con el tipo de red social donde se comparte la noticia (abierta o cerrada) y con el tipo de noticia (dura o blanda).

H2. La frecuencia de consumo de noticias habitual influirá en el grado de lectura de las noticias compartidas.

H3. El nivel de disfrute de las noticias influirá en el grado de su lectura.

H4. La evaluación de las noticias (afecto positivo y utilidad) será diferente en las noticias compartidas en las distintas redes. Así, las noticias evaluadas como más útiles pueden dirigirse a redes abiertas, de forma que ayuden a construir la imagen propia, mientras que las que generan más afecto van a redes cerradas.

En cuanto a este último aspecto, el afecto, cabe esperar que influya especialmente en aquellas plataformas que suelen servir para reforzar lazos ya existentes, como sucede con WhatsApp (Bano et al., 2019) y Facebook (Shane-Simpson et al., 2018).

H5. Los temas de las noticias que se consumen y se difunden serán distintos en las diferentes redes sociales. Así, las noticias duras tenderán a compartirse más en redes cerradas, en las que se genera cierta autocensura para evitar las polémicas (Marwick; Boyd, 2011; Kwon; Moon; Stefanone, 2015).

\section{Metodología}

Siguiendo las recomendaciones de Witschge et al. (2018) este estudio no considera como unidad de análisis los individuos sino las situaciones por las que atraviesan dado que un usuario puede reaccionar de diferentes modos y estar motivado por distintos factores en distintos momentos. Para ello se utiliza un método de muestreo de experiencias (MME), que es habitual en análisis de la actividad de usuarios de redes sociales (como el empleado en Hall, 2018; Trieu, et al., 2019). Este método implica que un grupo de participantes voluntarios debe contestar, en distintos momentos temporales, a una serie de preguntas sobre alguna actividad que han realizado o alguna experiencia por la que han atravesado (Kubey; Larson; Csikszentmihalyi, 1996).

El universo buscado para la muestra corresponde a la población española de jóvenes y jóvenes adultos (18-39 años) puesto que sus actitudes de uso de las noticias pueden marcar los patrones de las futuras tendencias de distribución social y consumo de noticias (Bobkowski, 2015). El reclutamiento de los 300 voluntarios iniciales participantes en el 
estudio corrió a cargo de la empresa demoscópica Societae. Se partió de un muestreo aleatorio estratificado, aunque la muestra final guarda algunas descompensaciones respecto al universo de estudio, tal y como se menciona en el apartado de limitaciones del estudio.

Esos momentos temporales fueron tres: 16 de noviembre (sábado), 24 de noviembre (domingo) y 2 de diciembre de 2019 (lunes). El reparto de estas fechas responde a un criterio de equilibrio, para redistribuir de manera equidistante las tres oleadas durante el período considerado para obtener así un mayor número de experiencias distintas entre sí. Del mismo modo se ha escogido preferentemente fechas cercanas al fin de semana por considerar que al ser un período de ocio los participantes tendrían mayor tiempo para dedicar a la lectura de noticias. Como ya se ha señalado, este trabajo toma como objeto las situaciones, no los sujetos. Por ello, para aumentar la información acerca de situaciones por las que atraviesan estos sujetos se les ha inquirido tres veces.

En cada una de las tres oleadas se envió a todos los participantes un correo electrónico con preguntas referidas a la última noticia que hubieran compartido en redes sociales, mensajería instantánea o cualquier otro medio. Estas preguntas fueron comunes y se repitieron en los tres momentos del estudio. Es decir, cada sujeto pudo responder hasta en tres ocasiones a las preguntas referidas a esa última noticia que hubiera compartido con algún contacto. Se indicó a los participantes que, si desde la anterior oleada no habían compartido una nueva noticia, no debían contestar al correo. De este modo se planteaba evitar la duplicidad de información acerca de una misma experiencia.

\subsection{Medidas y herramientas de recogida de información}

Los participantes debieron evaluar cada una de las noticias que compartieron.

El afecto positivo $(\alpha=0,86)$ se calculó como el sumatorio de las respuestas a cuatro preguntas tipo Likert: (1=mínimo acuerdo, 5=máximo acuerdo) “Me ha gustado el contenido", "He disfrutado el contenido", "El contenido es positivo", "El contenido es entretenido".

Para medir la utilidad $(\alpha=0,85)$ se plantearon cinco preguntas tipo Likert en una escala de 1 a 5: "El contenido es valioso (en general, para mí, para mis amigos o para la sociedad)", "El contenido es bueno y de calidad", "El contenido es útil", "El contenido me ha ayudado a estar informado" y "El contenido es relevante para mi vida". El orden de las preguntas de este bloque se aleatorizó en cada cuestionario y para cada participante.

De manera concreta, los participantes debieron indicar el modo en el que accedieron a la información que compartieron posteriormente (tabla 3), la profundidad con la que leyeron la noticia antes de compartirla (en profundidad, superficialmente o sólo el titular, ver tabla 4) y el entorno o plataforma en el que compartió la noticia (tabla 5).

Los participantes proporcionaron asimismo el enlace de la noticia compartida. Se especificaba un concepto amplio de "noticia", que no exigía que hubiese sido publicada por un medio tradicional o de masas. Si la noticia no incluía un enlace se pidió a los usuarios que indicaran el titular de la noticia. Posteriormente, un investigador del equipo categorizó estas noticias de acuerdo con los temas definidos por Kilgo et al., (2018). Finalmente se agruparon los bloques de preguntas en cuatro grandes temas -noticias duras, noticias blandas, cultura y otros- (tabla 7).

Adicionalmente, en la primera oleada se incluyó un bloque de preguntas específico sobre datos personales de los sujetos para su caracterización sociodemográfica: edad, género, provincia de residencia, máximo nivel educativo logrado y nivel de ingresos mensuales. Además de estas preguntas sociodemográficas los sujetos también tuvieron que indicar su nivel de disfrute de las noticias en general (pregunta Likert de escala 1 a 5, "Disfruto estando al tanto de las noticias y de la actualidad”). También se registró el consumo habitual de noticias: (“¿Con qué frecuencia ve, escucha o lee noticias, ya sea en la televisión, en la radio, en la prensa o mediante internet?") que presentaba una escala del 1 al 8 con los siguientes niveles: 1: Nunca, 2: Muy esporádicamente, 3: Alguna vez al mes, 4: Una vez a la semana, 5: Varias veces a la semana, 6: Una vez al día, 7: Varias veces al día, 8: Prácticamente cada hora.

Las preguntas del cuestionario se incluyen como Anexo I del estudio.

\subsection{Análisis de los datos}

El tratamiento estadístico de los datos se ha llevado a cabo mediante el software $R$. Para contrastar las hipótesis que plantean relaciones entre variables categóricas $(\mathrm{H} 1, \mathrm{H} 3$ y H5) se ha empleado la prueba de independencia de chi cuadrado $\left(\chi^{2}\right)$ de Pearson. En esta prueba el valor $\chi^{2}$ indica hasta qué punto existe relación entre las categorías. Cuanto más se aleje ese índice de 0 , mayor es la probabilidad de que las categorías relacionadas sean independientes entre sí. Para cada test se ofrece también la información acerca del número de casos considerados en cada ocasión $(N)$ y los grados de libertad, es decir, las distintas asociaciones posibles entre categorías. La prueba también ofrece un valor de significatividad (p) para el contraste de la hipótesis. Cuanto más se acerque ese valor $p$ a 0 , más certeza existe de que haya una asociación estadísticamente significativa entre las variables consideradas. El umbral de significatividad de este valor se sitúa en 0,05. El resultado de la prueba chi cuadrado de Pearson se indica en el apartado de resultados ofreciendo primero entre paréntesis el grado de libertad y el número total $(N)$ de observaciones sobre las que se calcula la prueba, a continuación se ofrece el valor de chi cuadrado y a continuación el valor de $p$ (siguiendo las recomendaciones de la American Psychological Association, sólo se ofrecen los valores decimales). Así, la expresión $\chi^{2}(8, N=830)=8,78, p=0,36$ 
indica que la prueba chi cuadrado se ha calculado sobre un conjunto de 830 observaciones pertenecientes a ocho combinaciones de categorías, cuyo resultado es 8,78 y cuya significatividad es 0,36 , lo que conlleva desechar la hipótesis.

Las tablas 8 y 9 muestran la distribución observada y la distribución teórica para cada una de las relaciones de categorías planteadas en las hipótesis

Para comprobar la hipótesis referida a la existencia de diferencias de valores entre dos o más categorías (H2, H4) se han utilizado tests de contraste no paramétrico. Se ha optado por una prueba no paramétrica dado que las variables cuantitativas se midieron en términos ordinales (escalas de Likert), no gaussianos.

En los supuestos (hipótesis 4) en los que se comparan sólo dos grupos (noticias compartidas en un tipo de red social o plataforma determinada frente a las que se comparten en otro tipo de red social o entorno concreto) se ha empleado la prueba U de Mann Whitney. Esta prueba calcula también la significatividad de las diferencias encontradas con el cálculo de un valor $p$, cuyo umbral para determinar si se cumple la hipótesis se sitúa en valores iguales o inferiores a 0,05. El resultado de este valor se indica en el texto. Las tablas 11 y 12 reflejan los valores promedio obtenidos en cada conjunto comparado, la desviación estándar $(\sigma)$ de cada media y el número $(N)$ de observaciones pertenecientes a cada caso. Se ofrece también la diferencia entre las medias obtenidas entre ambos conjuntos. Sólo se ofrece esta información en aquellos casos en los que el valor $p$ arroja una diferencia significativa.

En aquellos supuestos en que las diferencias se comparaban entre más de tres grupos (por ejemplo, los niveles de lectura del artículo) se ha empleado un Anova (análisis de varianza) unidireccional (Kruskal-Wallis). Al igual que la prueba de Mann Whitney este test calcula un valor $\mathrm{p}$ que indica diferencias significativas entre los conjuntos considerados cuando dicho índice alcanza o se sitúa por debajo de 0,05. Si el valor $\mathrm{p}$ se sitúa en valores significantes resulta necesario aplicar una prueba post hoc para ubicar los grupos entre los cuales se produce esa diferencia significativa. Así, la prueba Dwass-Steel-Critchlow-Fligner (tabla 10) calcula un valor $\mathrm{p}$ para las diferencias bilaterales entre cada uno de los conjuntos observados. De nuevo, si ese valor $p$ es igual o inferior a 0,05, las diferencias entre las dos categorías se pueden considerar significativas. La tabla 10 ofrece ese valor $p$, así como la diferencia entre el promedio de cada grupo comparado.

\section{Resultados}

\subsection{Descriptivos}

\subsubsection{Participantes}

La muestra final quedó reducida a 279 sujetos después de que 21 de ellos no contestaran al cuestionario inicial del estudio. La media de edad de los participantes se sitúa en 27,9 años $(\sigma=6,08)$. La tabla 1 recoge el resto de las características sociodemográficas de la muestra.

Los participantes fueron reclutados de 33 provincias españolas, aunque las regiones más representadas fueron Madrid, Granada y Barcelona (tabla 2).

Tabla 1. Características sociodemográficas de la muestra

\begin{tabular}{|c|c|c|c|}
\hline & & \multirow{2}{*}{$\begin{array}{c}\mathbf{n} \\
109\end{array}$} & \multirow{2}{*}{$\begin{array}{c}\% \\
39,07\end{array}$} \\
\hline \multirow{3}{*}{ Género } & Masculino & & \\
\hline & Femenino & 167 & 59,86 \\
\hline & Prefiero no decirlo & 3 & 1,08 \\
\hline \multirow{5}{*}{ Educación } & Básica & 5 & 1,79 \\
\hline & Secundaria & 40 & 14,34 \\
\hline & Profesional & 71 & 25,45 \\
\hline & Universitaria & 162 & 58,07 \\
\hline & Doctorado & 1 & 0,36 \\
\hline \multirow{12}{*}{$\begin{array}{l}\text { Ingresos } \\
\text { mensuales }\end{array}$} & Sin ingresos & 9 & 3,23 \\
\hline & $€ 0<300$ & 6 & 2,15 \\
\hline & $€ 301<600$ & 14 & 5,02 \\
\hline & $€ 601<900$ & 17 & 6,09 \\
\hline & $€ 0<1.200$ & 36 & 12,90 \\
\hline & $€ 1.201<2.400$ & 67 & 24,01 \\
\hline & $€ 1.801<2.400$ & 46 & 16,49 \\
\hline & $€ 2.401<3.000$ & 38 & 13,62 \\
\hline & $€ 3.001<4.500$ & 29 & 10,39 \\
\hline & $€ 4.501<6.000$ & 7 & 2,51 \\
\hline & $€>6.000$ & 2 & 0,72 \\
\hline & Prefiero no decirlo & 8 & 2,87 \\
\hline
\end{tabular}

Tabla 2. Distribución de los participantes por provincia

\begin{tabular}{|c|c|c|c|c|c|c|c|c|}
\hline Provincia & $\mathbf{n}$ & $\%$ & Provincia & $\mathbf{n}$ & $\%$ & Provincia & n & $\%$ \\
\hline A Coruña & 18 & 6,2 & Castellón & 1 & 0,3 & Navarra & 1 & 0,3 \\
\hline Álava & 1 & 0,3 & Córdoba & 5 & 1,7 & Palencia & 1 & 0,3 \\
\hline Albacete & 12 & 4,1 & Cuenca & 2 & 0,7 & Pontevedra & 8 & 2,8 \\
\hline Alicante & 1 & 0,3 & Granada & 62 & 21,4 & Salamanca & 5 & 1,7 \\
\hline Almería & 4 & 1,4 & Guadalajara & 2 & 0,7 & Sevilla & 4 & 1,4 \\
\hline Asturias & 1 & 0,3 & Guipúzcoa & 2 & 0,7 & Tarragona & 1 & 0,3 \\
\hline Badajoz & 2 & 0,7 & Jaén & 2 & 0,7 & Toledo & 2 & 0,7 \\
\hline Baleares & 2 & 0,7 & León & 1 & 0,3 & Valencia & 4 & 1,4 \\
\hline Barcelona & 31 & 10,7 & Madrid & 75 & 25,9 & Valladolid & 5 & 1,7 \\
\hline Burgos & 6 & 2,1 & Málaga & 5 & 1,7 & Vizcaya & 13 & 4,5 \\
\hline Cádiz & 3 & 1,0 & Murcia & 6 & 2,1 & Zamora & 2 & 0,7 \\
\hline
\end{tabular}


La media de consumo de noticias de los participantes, medida en la escala referida anteriormente en la que 1 equivale a nunca y 8 a prácticamente cada hora se sitúa en $2,59(\sigma=1,12)$. En cuanto al nivel de disfrute de los usuarios del consumo de noticas, el promedio es de 3,82 $(\sigma=0,943)$, en una escala del 1 al 5 .
El consumo social de noticias varía entre el lector incidental, que se conecta por ocio o entretenimiento y el lector rutinario, que monitorea la Red en busca de noticias de su interés

Respecto a la evaluación de las noticias compartidas, el afecto positivo alcanza un promedio de 13,72 ( $\sigma=4,44)$ sobre 20 y la utilidad percibida, una media de 18,82 ( $\sigma=4,39)$ sobre 25.

\subsubsection{Experiencias recogidas}

En el conjunto de las tres oleadas se recogió en total información acerca de 830 noticias compartidas, las experiencias que como se ha señalado constituyen el material sobre el que se construye el análisis.

La exposición incidental constituyó la principal vía de acceso a las noticias compartidas (tabla 3)

Tabla 3. Vías de acceso a la información

\begin{tabular}{|c|c|c|}
\hline & \\
\hline & $\mathbf{n}$ & $\%$ \\
\hline 1. Estaba navegando en Internet, sin buscar nada en particular, y me he encontrado con ella & 194 & 23,37 \\
\hline 2. La he encontrado en una web que visito habitualmente para enterarme de las últimas noticias & 120 & 14,46 \\
\hline 3. Estaba buscando información sobre el tema (en Google u otro buscador como Bing) y he encontrado la noticia & 69 & 8,31 \\
\hline $\begin{array}{l}\text { 4. Tengo instalada la app del medio de mi teléfono móvil y me ha avisado mediante una notificación automática o } \\
\text { personalizada }\end{array}$ & 13 & 1,57 \\
\hline $\begin{array}{l}\text { 5. Me he enterado por un servicio de alertas del medio al que estoy suscrito, por una newsletter, o por otros medios } \\
\text { como el canal de Telegram del medio }\end{array}$ & 18 & 2,17 \\
\hline $\begin{array}{l}\text { 6. Algún amigo o contacto me ha facilitado el enlace o me ha avisado de la noticia por correo electrónico, mensajería } \\
\text { móvil o etiquetándome en redes sociales }\end{array}$ & 122 & 14,70 \\
\hline $\begin{array}{l}\text { 7. Estaba viendo mis redes sociales y he encontrado la noticia, en un mensaje que alguien ha publicado pero que no } \\
\text { me mencionaba a mí directamente, ni en un grupo del que formo parte. }\end{array}$ & 255 & 30,72 \\
\hline 8. Otro & 39 & 4,70 \\
\hline
\end{tabular}

Las opciones 1,6 y 7 se agregaron en la categoría "exposición incidental", las opciones 2, 4 y 5, en la "vigilancia rutinaria" y la 3 en "consumo directo". En el caso de la opción 8 (39), "otro", los participantes debieron especificar y ofrecer más información al respecto. Esas respuestas fueron revisadas por un investigador y 30 pudieron ser agregadas en las categorías previas. De las nueve restantes, seis hacían referencia a que el propio usuario había sido el autor de la noticia (creando, por ejemplo, un post en un blog) y las otras tres restantes no encajaron en ninguna otra categoría. Finalmente, estas nueve respuestas no entraron en el análisis. Así, la exposición por

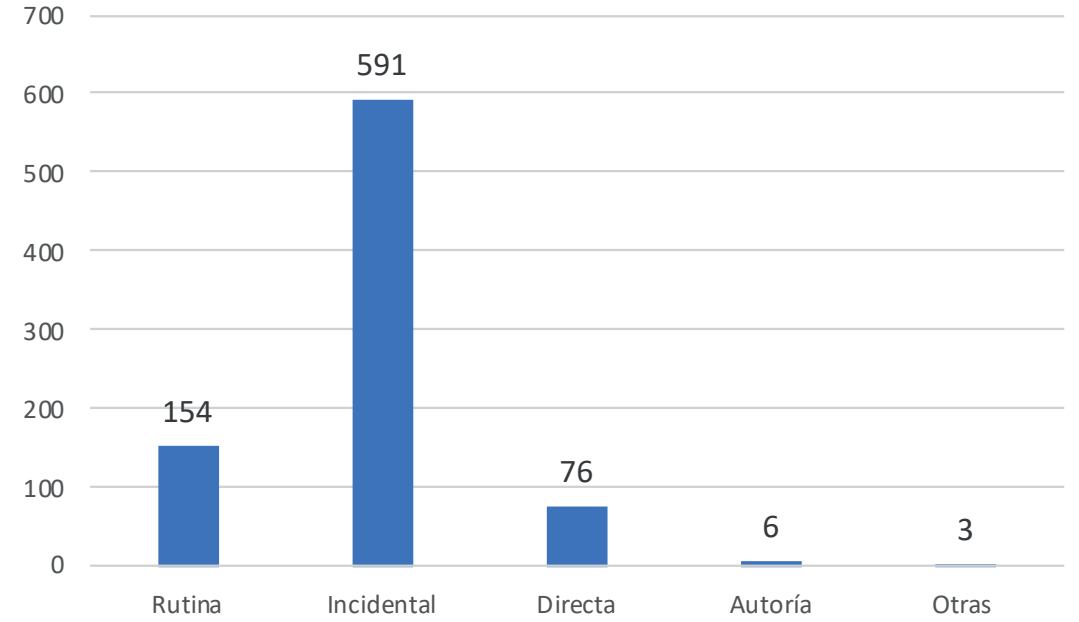

Gráfico 1. Frecuencia de categorías de acceso a la información rutina supuso el $28,55 \%$ de respuestas, la incidental el $71,20 \%$ y la directa el $9,16 \%$ (gráfico 1).

En la mayor parte de las ocasiones los usuarios expresan haber leído la noticia en profundidad antes de compartirla con sus contactos (tabla 4).

Tabla 4. Frecuencia de profundidad de las lecturas compartidas

\begin{tabular}{|c|c|c|}
\hline He visitado el enlace al texto completo y he leído la noticia en profundidad & 569 & 68,55 \\
\hline He visitado el enlace al texto completo, pero he leído la noticia superficialmente, por encima & 177 & 21,32 \\
\hline Sólo he leído el titular o la vista previa de la noticia, no he visitado el enlace al texto de la noticia & 84 & 10,12 \\
\hline
\end{tabular}


Las noticias fueron compartidas de manera preferente en una red social de manera abierta o bien en un grupo privado de mensajería instantánea (tabla 5).

Tabla 5. Plataformas en las que se compartieron las noticias

1. En alguna red social (Twitter, Facebook, Instagram...) como una publicación para que la puedan ver todos mis contactos en general

2. En redes sociales, pero me he dirigido a una persona concreta, etiquetándole en Facebook o Instagram o mencionándole en Twitter. Aun así, el mensaje era público y lo podían ver todos mis contactos

3. En un grupo privado o comunidad restringida de Facebook o cualquier otra red social, de modo que solo lo puedan ver los miembros de ese grupo o comunidad

4. En un grupo privado de WhatsApp, Line, Telegram o Facebook Messenger

5. Se lo he enviado a un contacto concreto mediante mensajería instantánea (WhatsApp, Line, Telegram o Facebook Messenger)

6. Por correo electrónico, con una persona o grupo de personas determinado

7. Por correo electrónico, de manera masiva a un gran grupo de personas (más de 50)

8. Otro

\begin{tabular}{|c|c|}
\hline $\mathbf{n}$ & $\%$ \\
\hline 414 & 49,88 \\
\hline 25 & 3,01 \\
\hline 12 & 1,45 \\
\hline 252 & 30,36 \\
\hline 112 & 13,49 \\
\hline 6 & 0,72 \\
\hline 0 & 0 \\
\hline 9 & 1,09 \\
\hline
\end{tabular}

\begin{tabular}{|c|c|c|c|}
\hline & & $\mathbf{n}$ & $\%$ \\
\hline & $\begin{array}{l}\text { 1. En alguna red social (Twitter, Facebook, Instagram...) como una publicación para que la puedan ver } \\
\text { todos mis contactos en general }\end{array}$ & 414 & 49,88 \\
\hline $\begin{array}{l}\text { RAA } \\
(53,37 \%)\end{array}$ & $\begin{array}{l}\text { 2. En redes sociales, pero me he dirigido a una persona concreta, etiquetándole en Facebook o Instagram } \\
\text { o mencionándole en Twitter. Aun así, el mensaje era público y lo podían ver todos mis contactos }\end{array}$ & 25 & 3,01 \\
\hline & Otras RAA & 4 & 0,48 \\
\hline & $\begin{array}{l}\text { 3. En un grupo privado o comunidad restringida de Facebook o cualquier otra red social, de modo que } \\
\text { solo lo puedan ver los miembros de ese grupo o comunidad }\end{array}$ & 12 & 1,45 \\
\hline & 4. En un grupo privado de WhatsApp, Line, Telegram o Facebook Messenger & 252 & 30,36 \\
\hline $\begin{array}{l}\text { RCS } \\
(46,14 \%)\end{array}$ & $\begin{array}{l}\text { 5. Se lo he enviado a un contacto concreto mediante mensajería instantánea (WhatsApp, Line, Telegram o } \\
\text { Facebook Messenger) }\end{array}$ & 112 & 13,49 \\
\hline & 6. Por correo electrónico, con una persona o grupo de personas determinado & 6 & 0,72 \\
\hline & 7. Por correo electrónico, de manera masiva a un gran grupo de personas (más de 50) & 0 & 0,00 \\
\hline & Otras RCS & 1 & 0,12 \\
\hline Otros & 8. Otros & 4 & 0,48 \\
\hline
\end{tabular}

Tabla 6. Noticias compartidas por plataforma

\begin{tabular}{|l|c|}
\hline Plataforma & Frecuencia \\
\hline Facebook & 362 \\
\hline Twitter & 59 \\
\hline Instagram & 30 \\
\hline Otros & 95 \\
\hline WhatsApp & 88 \\
\hline Telegram & 2 \\
\hline Linkedln & 3 \\
\hline Facebook Messenger & 4 \\
\hline
\end{tabular}

Las opciones 1-2 se agruparon como RAA y las 3-6 se tomaron como RCS. La opción 8 ("Otros") requería que el usuario ofreciese más información al respecto. Con esta explicación de los participantes las respuestas obtenidas se recodificaron de forma que una se categorizó como RCS y cuatro como RAA. Las otras cuatro no ofrecían suficiente información para poder incluirse en uno de los dos grupos.
Tabla 7. Tipo de noticia compartida

\begin{tabular}{|c|c|c|c|}
\hline & Tema & $\mathbf{n}$ & $\%$ \\
\hline \multirow{8}{*}{ Noticias duras } & Relaciones internacionales & 45 & 5,74 \\
\hline & Ejército / Defensa & 2 & 0,26 \\
\hline & Gobierno & 102 & 13,01 \\
\hline & Crimen / Sucesos & 128 & 16,33 \\
\hline & Economía / Negocios / Finanzas & 38 & 4,85 \\
\hline & Derechos civiles & 28 & 3,57 \\
\hline & Medio ambiente & 63 & 8,04 \\
\hline & Religión & 3 & 0,38 \\
\hline \multirow{3}{*}{ Noticias blandas } & Deportes & 46 & 5,87 \\
\hline & Entretenimiento & 96 & 12,24 \\
\hline & Estilo de vida / Salud & 67 & 8,55 \\
\hline \multirow{2}{*}{ Cultura } & Educación & 69 & 8,80 \\
\hline & Ciencia / Tecnología & 25 & 3,19 \\
\hline \multirow[t]{2}{*}{ Otros } & Otros & 72 & 9,18 \\
\hline & Total & 784 & 100 \\
\hline
\end{tabular}

En este sentido los usuarios que escogieron las opciones 1 a 5 pudieron indicar además la plataforma concreta en la que compartieron esa noticia. De este modo Facebook se sitúa como la plataforma preferida para compartir noticias (tabla $6)$. 
Respecto al tema de las noticias, se compartieron más frecuentemente noticias acerca de crímenes y sucesos, sobre política nacional y sobre entretenimiento (tabla 7).

La muestra de noticias compartidas según tema queda compuesta por 784 noticias en lugar de las 830 de la muestra global porque 46 ítems quedaron fuera del análisis, dado que no ofrecieron suficiente información como para asignarles una categoría. Ello implica que la $N$ sobre la que se calculan las hipótesis que implican la categorización de las noticias (1 y 5) es de 784 en lugar de 830.

\subsection{Contraste de hipótesis}

\subsubsection{Relación entre tipo de exposición y grado de lectura, red social donde se comparte la noticia y tipo de noticia} (H1).

Los resultados del chi cuadrado de Pearson refutan la hipótesis de que el tipo de exposición de la noticia se asocie al grado de profundidad de la lectura de la noticia compartida, ya que el valor $p$ obtenido supera el estándar de 0,05: $\chi^{2}(8$, $N=830)=8,78, p=0,36$.

Del mismo modo, la relación entre profundidad de lectura de la noticia y el tema de la noticia tampoco alcanza niveles suficientes de significatividad: $\chi^{2}(6, N=784)=7,74, p=0,258$.

Por el contrario, el chi cuadrado de Pearson sí que ha arrojado resultados positivos $\left[\chi^{2}(8, N=830)=117,03, p<, 001\right]$ para la relación entre el tipo de exposición y el tipo de red social en el que se comparte la noticia. Como se detalla en la tabla 8, la frecuencia de la exposición directa en el caso de noticias encontradas por vía directa y por rutina están por encima de la distribución teórica si no hubiera relación entre ambas variables. Del mismo modo, la frecuencia observada en el caso de noticias compartidas en RAA encontradas por exposición incidental superan también la distribución teórica.

Tabla 8. Distribución de casos observada y teórica según vía de exposición a la noticia y tipo de red social donde se comparte

\begin{tabular}{|c|c|c|c|c|c|c|c|}
\hline \multirow[b]{2}{*}{$\begin{array}{l}\text { Tipo de red } \\
\text { social }\end{array}$} & \multirow[b]{2}{*}{ Distribución } & \multicolumn{5}{|c|}{ Exposición a la noticia } & \multirow[b]{2}{*}{ Total (filas) } \\
\hline & & Otros & Autoría & Directa & Incidental & Rutina & \\
\hline \multirow{2}{*}{ Otras } & Observada & 1 & 0 & 0 & 1 & 2 & 4 \\
\hline & Teórica & 0,014 & 0,029 & 0,366 & 2,848 & 0,742 & \\
\hline \multirow{2}{*}{ Abierta } & Observada & 2 & 6 & 21 & 352 & 62 & 443 \\
\hline & Teórica & 1,601 & 3,202 & 40,564 & 315,437 & 82,195 & \\
\hline \multirow{3}{*}{ Cerrada } & Observada & 0 & 0 & 55 & 238 & 90 & 383 \\
\hline & Teórica & 1,384 & 2,769 & 35,070 & 272,714 & 71,063 & \\
\hline & $\begin{array}{l}\text { Total observadas } \\
\text { (columnas) }\end{array}$ & 3 & 6 & 76 & 591 & 154 & 830 \\
\hline
\end{tabular}

También se ha observado una relación significativa $\left[\chi^{2}(12, N=784)=24,08, p=0,016\right]$ entre el tipo de exposición a la noticia y el tema de las noticias. Concretamente se ha identificado que el consumo directo se relaciona con una mayor presencia de noticias duras. Por el contrario, la exposición incidental, se vincula con una menor frecuencia de este tipo de información (tabla 9). Este dato implica que el consumo de noticias duras se relaciona con el consumo directo.

Tabla 9. Distribución de casos observada y teórica según vía de acceso a la noticia y tema de la noticia

\begin{tabular}{|c|c|c|c|c|c|c|}
\hline Vía de acceso & Distribución & Blandas & Cultura & Duras & Otras & Total (filas) \\
\hline \multirow{2}{*}{ Otras } & Observada & 1 & 0 & 0 & 0 & 1 \\
\hline & Teórica & 0,267 & 0,120 & 0,522 & 0,092 & \\
\hline \multirow{2}{*}{ Autoría } & Observada & 0 & 2 & 3 & 0 & 5 \\
\hline & Teórica & 1,333 & 0,599 & 2,608 & 0,459 & \\
\hline \multirow{2}{*}{ Directa } & Observada & 12 & 4 & 55 & 5 & 76 \\
\hline & Teórica & 20,260 & 9,112 & 39,648 & 6,980 & \\
\hline \multirow{2}{*}{ Incidental } & Observada & 158 & 74 & 272 & 56 & 560 \\
\hline & Teórica & 149,286 & 67,143 & 292,143 & 51,429 & \\
\hline \multirow{3}{*}{ Rutina } & Observada & 38 & 14 & 79 & 11 & 142 \\
\hline & Teórica & 37,855 & 17,026 & 74,079 & 13,041 & \\
\hline & $\begin{array}{l}\text { Total observadas } \\
\text { (columnas) }\end{array}$ & 209 & 94 & 409 & 72 & 784 \\
\hline
\end{tabular}




\subsubsection{Relación entre frecuencia de consumo de noticias y grado de lectura de las noticias compartidas (H2)}

El resultado del Anova (análisis de varianza) unidireccional (Kruskal-Wallis) para las diferencias entre el grado de lectura de las noticias compartidas en función de la frecuencia de consumo de noticias no alcanza un valor significativo [ $\chi^{2}(6$, $N=830)=9,95, p=0,127$ ], lo que lleva a refutar la hipótesis de que el hábito de consumo de noticias conlleve una lectura en mayor o menor profundidad de las noticias que comparten los sujetos.

\subsubsection{Relación entre el nivel de disfrute de las noticias y grado de lectura de las noticias compartidas (H3)}

La prueba Anova (análisis de varianza) unidireccional (Kruskal-Wallis) obtiene un valor $\mathrm{p}$ situado dentro del umbral de significatividad $\left[\chi^{2}(2, N=830)=6,06, p=0,048\right]$, lo que indica que al menos las diferencias entre dos categorías consideradas son suficientemente relevantes como para que no se deban al azar. Los resultados de la prueba post-hoc Dwass-Steel-Critchlow-Fligner indican que a mayor grado de disfrute existe una mayor tendencia a leer la noticia en profundidad en tanto que los sujetos con niveles de disfrute más elevados obtienen un promedio de grado de lectura mayor que aquellos que se ubican en las posiciones inferiores de disfrute del consumo de noticias. Estas diferencias son significativas entre aquellos individuos que se sitúan en los valores extremos de la escala de disfrute de noticias (tabla 10).

4.2.4. Diferencias entre evaluación de las noticias según la red social en la que se comparten (H4)

La prueba de Mann Whitney ha indicado diferencias significativas en la evaluación obtenida por las noticias compartidas en distintos entornos. Concretamente, las noticias que se comparten en RAA son evaluadas de manera significativa $(p<0,001)$ con una mayor afectividad positiva (tabla 11). De manera simétrica, las noticias que se comparten en RCS muestran una diferencia significativa $(p<0,001)$ en un sentido opuesto. Es decir, las noticias que se comparten en RCS se evalúan con una menor carga afectiva. Las diferencias señaladas en el entorno general de las redes sociales abiertas asimétricas se encuentran también en el entorno concreto de Facebook $(p<0,001)$. No obstante, no se encuentran diferencias significativas en otros ejemplos de RAA como Twitter o Instagram, debido quizá a que el número de noticias compartidas en la muestra es excesivamente reducido.

La faceta de Facebook como un vehículo para la difusión de noticias afectivas se confirma con el hallazgo de que, además, estadísticamente las noticias que los usuarios encuentran en Facebook tienen significativamente $(p<0,001)$, de acuerdo con la prueba de Mann-Whitney, una mayor carga afectiva (tabla 12).

Respecto a la utilidad, las noticias compartidas en Facebook también muestran una diferencia significativa Tabla 10. Diferencias entre el grado de lectura de acuerdo con el nivel de disfrute de las noticias

\begin{tabular}{|c|c|c|c|}
\hline \multicolumn{4}{|c|}{ Post-hoc Dwass-Steel-Critchlow-Fligner } \\
\hline \multicolumn{2}{|c|}{ Nivel de disfrute } & Diferencia promedio & p \\
\hline 1 & 2 & $-2,11$ & 0,295 \\
\hline 1 & 3 & $-3,08$ & 0,075 \\
\hline 2 & 3 & $-1,42$ & 0,576 \\
\hline
\end{tabular}

Tabla 11. Comparación de afectividad positiva de las noticias

\begin{tabular}{|c|c|c|}
\hline & \multicolumn{2}{|c|}{ Noticias compartidas en RAA } \\
\hline & No & Sí \\
\hline Media & 13,125 & 14,255 \\
\hline$\sigma$ & 4,335 & 4,471 \\
\hline $\mathrm{N}$ & 391 & 439 \\
\hline \multirow[t]{3}{*}{ Diferencia de medias } & \multicolumn{2}{|c|}{1,130} \\
\hline & \multicolumn{2}{|c|}{ Noticias compartidas en RCS } \\
\hline & No & Sí \\
\hline Media & 14,241 & 13,115 \\
\hline$\sigma$ & 4,473 & 4,330 \\
\hline $\mathrm{N}$ & 448 & 382 \\
\hline \multirow[t]{3}{*}{ Diferencia de medias } & \multicolumn{2}{|c|}{1,126} \\
\hline & \multicolumn{2}{|c|}{ Noticias compartidas en Facebook } \\
\hline & No & Sí \\
\hline Media & 13,102 & 14,393 \\
\hline$\sigma$ & 4,345 & 4,451 \\
\hline $\mathrm{N}$ & 431 & 399 \\
\hline Diferencia de medias & \multicolumn{2}{|c|}{1,291} \\
\hline
\end{tabular}

Tabla 12. Comparación de la afectividad de las noticias encontradas en Facebook

\begin{tabular}{|l|c|c|}
\hline Encontradas en Facebook & No & Sí \\
\hline Media & 13,400 & 14,438 \\
\hline$\sigma$ & 4,428 & 4,394 \\
\hline N & 572 & 258 \\
\hline Diferencia de medias & \multicolumn{2}{|c|}{1,038} \\
\hline
\end{tabular}

$(p<0,001)$, en el sentido de que las noticias que se comparten en esta red social obtienen una valoración más alta (promedio=19,398, $\sigma=4,324$ ) que las que se comparten en otros entornos (promedio=18,306, $\sigma=4,399$ ). El resultado de la prueba de Mann-Whitney no ha resultado significativa para el resto de tipos de red social y de plataformas concretas.

\subsubsection{Diferencias entre temas de noticias y red social en la que se comparte (H5)}

De acuerdo al chi cuadrado de Pearson no se ha encontrado ninguna relación entre el tipo de noticias ni en el tipo de red social donde se comparte la noticia en los casos de

- $\operatorname{RAA} \chi^{2}(3, N=784)=4,77, \mathrm{p}=0,190$

- $\operatorname{RCS}(3, N=784)=6,70, p=0,072$

- Instagram $\chi^{2}(3, N=784)=2,21, p=0,530$

- WhatsApp $\chi^{2}(3, N=784)=0,50, p=0,918$

con lo que se concluye que estas categorías son independientes entre sí. 
Por el contrario, el valor $\mathrm{p}$ ha resultado significativo para la relación entre temas de noticias y dos redes sociales: Twitter y Facebook. En el caso de Twitter el chi cuadrado de Pearson arroja unos valores de $\chi^{2}(3, N=784)=0,50$, $\mathrm{p}=0,006$ y en la asociación con Facebook el resultado es $\chi^{2}(3, N=784)=8,03, p=0,045$.
Las exposiciones a hechos noticiosos que requieren un mayor esfuerzo suelen compartirse en redes cerradas. Mientras en las redes abiertas se tiende a compartir información más superficial

En el caso de Twitter las mayores diferencias entre la distribución esperada y la observada se dan en una menor presencia de noticias duras respecto a lo esperado y, como contrapartida, una aparición de noticias blandas que supera la distribución teórica (tabla 13). En otras palabras, en Twitter se comparten más frecuentemente noticias blandas que noticias duras.

Tabla 13. Distribución de casos observada y teórica según noticias compartidas en Twitter y tema de la noticia

\begin{tabular}{|c|c|c|c|c|c|c|}
\hline Compartido en Twitter & Distribución & Blandas & Cultura & Duras & Otras & Total (filas) \\
\hline \multirow{2}{*}{ No } & Observada & 183 & 84 & 386 & 70 & 723 \\
\hline & Teórica & 192,739 & 86,686 & 377,177 & 66,398 & \\
\hline \multirow{3}{*}{ Sí } & Observada & 26 & 10 & 23 & 2 & 61 \\
\hline & Teórica & 16,261 & 7,314 & 31,823 & 5,602 & \\
\hline & $\begin{array}{l}\text { Total observadas } \\
\text { (columnas) }\end{array}$ & 209 & 94 & 409 & 72 & 784 \\
\hline
\end{tabular}

En el caso de las noticias compartidas en Facebook, las diferencias se encuentran en las noticias de cultura y las noticias blandas, que se sitúan respectivamente por encima y por debajo de la distribución teórica (tabla 14). Es decir, las noticias de cultura se comparten más habitualmente en Facebook en comparación con el resto de las plataformas y servicios.

Tabla 14. Distribución de casos observada y teórica según noticias compartidas en Facebook y tema de la noticia

\begin{tabular}{|c|l|l|c|c|c|c|}
\hline Compartido en Facebook & \multicolumn{1}{|c|}{ Distribución } & Blandas & Cultura & Duras & Otras \\
\hline \multirow{2}{*}{ No } & Observada & 121 & 38 & 218 & 39 \\
\cline { 2 - 7 } & Teórica & 110,898 & 49,878 & 217,020 & 38,204 \\
\hline \multirow{2}{*}{ Sí } & Observada & 88 & 56 & 191 & 33 \\
\cline { 2 - 7 } & Teórica & 98,102 & 44,122 & 36,796 & 709 \\
\hline
\end{tabular}

\section{Conclusiones}

Los resultados obtenidos permiten señalar, en primer lugar, que el tipo de red social ejerce una mayor influencia que el tipo de exposición en los comportamientos de los usuarios cuando consumen y difunden información en redes sociales.

Así, por un lado, se ha comprobado que no existe relación entre el tipo de exposición y el grado de lectura de la noticia. Sin embargo, sí que se ha verificado que existe relación con el tipo de red social en la que se comparte la noticia, de manera que la exposición rutinaria y el consumo directo suelen llevar a compartir en redes cerradas, mientras que la exposición incidental suele llevar a hacerlo en redes abiertas.

Este dato puede interpretarse en el sentido de que es más probable que las exposiciones que requieren mayor esfuerzo o están vinculadas a un mayor interés por el contenido se compartan en redes cerradas. Es decir, que cuando el lector realiza una búsqueda activa (consumo directo) o lleva a cabo una rutina de monitoreo, suele compartir esas noticias en entornos más privados y dirigidos a una audiencia más concreta. Mientras, en las redes abiertas se tiende a compartir información de carácter más superficial, con la que los usuarios simplemente "se topan".

Esta conclusión ratifica el hallazgo de Kümpel, Karnowski y Keyling (2015), quienes ya apuntaron a que el tipo de red era uno de los factores que determina qué clase de contenido se comparte. Por el contrario, supone una novedad respecto a los resultados recogidos por Thorson (2020), quien establecía que apenas había diferencias al consumir y difundir noticias por el hecho de encontrarse en Facebook (red social abierta) o WhatsApp (red cerrada).

Del mismo modo, esta conclusión presenta implicaciones interesantes al conectarla con la obtenida por Salaverría et al. (2020), quienes averiguaron que los bulos o informaciones falsas, relacionadas precisamente con noticias más relevantes, se comparten mucho más fácilmente en redes cerradas. Se abre así el interrogante de si los bulos se comparten prioritariamente en entornos cerrados por el tipo de información que contienen o si el motivo de esta dinámica está en que los usuarios encuentran esta información no tanto de manera incidental sino por rutina o es un tipo de contenido que buscan activamente.

En ese sentido, el hecho de que el tipo de exposición no influya de forma tan clara coincide con las conclusiones obtenidas por Park y Kaye (2020), pero supone una
El nivel de disfrute de las noticias influye en su grado de lectura 
novedad respecto a otros estudios previos como el de Fletcher y Nielsen (2018), quienes sí establecían esa incidencia más claramente, sobre todo en referencia a la exposición incidental, a la hora de consumir y compartir noticias en las redes sociales.

Asimismo, a la vista de los resultados obtenidos se ha refutado la $\mathrm{H} 2$, es decir, el nivel de consumo de noticias habitual no influye en el grado de lectura de los contenidos que se comparten. Esta conclusión también supone una novedad con respecto al estudio de Fletcher y Nielsen (2018), quieres hallaron que la frecuencia de consumo habitual sí que correlacionaba positivamente con el tipo de exposición y el grado de lectura, o al de Kümpel, Karnowski y Keyling (2015), quienes también establecían una relación entre el nivel de consumo y el grado de lectura.

En otro orden de cosas, se ha verificado la $\mathrm{H} 3$ y se ha comprobado que el nivel de disfrute de las noticias influye en s grado de lectura de las mismas. Así, cuando más se disfruta leyendo las noticias que se comparten, con mayor profundidad se leen.

Esta conclusión supone también una novedad frente a los resultados obtenidos por Fletcher y Nielsen (2018), quienes concluyeron que las características de los sujetos, y en concreto su edad, sí que influían de forma clara en sus patrones de comportamiento a la hora de consumir y difundir noticias. Este hallazgo puede estar condicionado por la naturaleza de nuestra muestra, ya que se trata de usuarios de una franja de edad comprendida por adultos y jóvenes adultos. Quizá los hábitos en franjas superiores o inferiores muestren características distintas en este sentido.

También se ha verificado la $\mathrm{H} 4$ y el hecho de que la evaluación de las noticias varía en función del tipo de red:

- las noticias compartidas en Facebook tienen más afecto y utilidad que las que se comparten en otros entornos;

- las noticias con mayor carga afectiva se comparten principalmente en redes sociales abiertas;

- las noticias con mayor percepción de utilidad se comparten fuera de Twitter.

Estas conclusiones ratifican las obtenidas por Fletcher y Nielsen (2018), quienes también encontraron una relación entre la utilidad de las noticias y una mayor probabilidad de que éstas fueran compartidas en Facebook frente a Twitter. Del mismo modo, se señala también que las redes sociales abiertas constituyen un escenario donde prima fundamentalmente el contenido afectivo.

Finalmente, existen diferencias en los temas de las noticias que se consumen y difunden en función de la red social (H5). De este modo, mientras que en Twitter se detecta una mayor preferencia hacia noticias blandas, en el resto de las redes sociales y entornos no se ha encontrado ninguna diferencia significativa. Esta última conclusión conecta con la obtenida por Salaverría et al., (2020) relativa a la distribución de contenidos, en concreto de bulos e informaciones falsas, que también encuentran mayor facilidad para ser diseminados en redes como WhatsApp.

Los hallazgos y conclusiones del estudio deben interpretarse con una serie de limitaciones en mente. En primer lugar, el universo estudiado sólo hace referencia a adultos entre 18 y 39 años, con lo que las características y rasgos detectados pueden no ser comunes a otros sectores de edad como los adolescentes o el público más maduro. Además, la población femenina, con estudios universitarios y con mayores niveles de ingresos resulta sobrerrepresentada en el conjunto de la muestra, lo que debe tenerse en cuenta a la hora de interpretar los resultados.

El estudio señala una serie de diferencias significativas que futuros estudios podrían someter a diseños experimentales para comprobar si existe una relación causal entre los factores comparados en este trabajo.

\section{Referencias}

Antunovic, Dunja; Parsons, Patrick; Cooke, Tanner R. (2018). “'Checking' and googling: Stages of news consumption among young adults". Journalism, v. 19, n. 5, pp. 632-648.

https://doi.org/10.1177/1464884916663625

Bakshy, Eytan; Rosenn, Itamar; Marlow, Cameron; Adamic, Lada (2012). "The role of social networks in information diffusion". In: Proceedings of the $21^{\text {st }}$ international conference on world wide web. New York, NY: ACM, pp. 519-528. https://doi.org/10.1145/2187836.2187907

Bano, Shehar; Cisheng, Wu; Khan, Ali-Nawaz; Khan, Nasser-Abbas (2019). “WhatsApp use and student's psychological well-being: Role of social capital and social integration". Children and youth services review, v. 103, pp. 200-208. https://doi.org/10.1016/j.childyouth.2019.06.002

Berger, Jonah (2011). "Arousal increases social transmission of information". Psychological science, v. 22, n. 7, pp. 891-893. https://doi.org/10.1177/0956797611413294

Bergström, Annika; Jervelycke-Belfrage, Maria (2018). "News in social media. Incidental consumption and the role of opinion leaders". Digital journalism, v. 6, n. 5, pp. 583-598.

https://doi.org/10.1080/21670811.2018.1423625 
Bobkowski, Piotr S. (2015). "Sharing the news: Effects of informational utility and opinion leadership on online news sharing". Journalism \& mass communication quarterly, v. 92, n. 2, pp. 320-345.

https://doi.org/10.1177/1077699015573194

Boczkowski, Pablo J.; Mitchelstein, Eugenia; Matassi, Mora (2018). "News comes across when I'm in a moment of leisure: Understanding the practices of incidental news consumption on social media". New media \& society, v. 20, n. 10, pp. 3523-3539.

https://doi.org/10.1177/1461444817750396

Bode, Leticia (2016). "Political news in the news feed: Learning politics from social media". Mass communication and society, v. 19, n. 1, pp. 24-48.

https://doi.org/10.1080/15205436.2015.1045149

Bright, Jonathan (2016). "The social news gap: How news reading and news sharing diverge". Journal of communication, v. 66, n. 3, pp. 343-365.

https://doi.org/10.1111/jcom.12232

Carlson, Matt (2016). “Embedded links, embedded meanings. Social media commentary and news sharing as mundane media criticism". Journalism studies, v. 17, n. 7, pp. 915-924.

https://doi.org/10.1080/1461670X.2016.1169210

Dafonte-Gómez, Alberto (2018). “News media and the emotional public sphere audiences as medium: Motivations and emotions in news sharing". International journal of communication, v. 12, pp. 2133-2152.

https://ijoc.org/index.php/ijoc/article/view/6790

Erdelez, Sandra (1999). "Information encountering: It's more than just bumping into information". Bulletin of the American Society for Information Science and Technology, v. 25, n. 3, pp. 26-29.

https://doi.org/10.1002/bult.118

Feezell, Jessica T. (2018). "Agenda setting through social media: The importance of incidental news exposure and social filtering in the digital era". Political research quarterly, v. 71, n. 2, pp. 482-494.

https://doi.org/10.1177/1065912917744895

Fletcher, Richard; Nielsen, Rasmus-Kleis (2018). "Are people incidentally exposed to news on social media? A comparative analysis". New media and society, v. 20, n. 7, pp. 2450-2468.

https://doi.org/10.1177/1461444817724170

Gil de Zúñiga, Homero; Weeks, Brian; Ardèvol-Abreu, Alberto (2017). "Effects of the news-finds-me perception in communication: Social media use implications for news seeking and learning about politics". Journal of computer-mediated communication, v. 22, n. 3, pp. 105-123.

https://doi.org/10.1111/jcc4.12185

Gonçalves, Jorge; Kostakos, Vassilis; Venkatanathan, Jayant (2013). "Narrowcasting in social media: Effects and perceptions". In: Proceedings of the 2013 IEEE/ACM international conference on advances in social networks analysis and mining, Asonam 2013, pp. 502-509.

https://doi.org/10.1145/2492517.2492570

Guallar, Javier; Suau, Jaume; Ruiz-Caballero, Carlos; Sáez, Albert; Masip, Pere (2016). "Re-dissemination of news and public debate on social networks". El profesional de la información, v. 25, n. 3, pp. 358-366.

https://doi.org/10.3145/epi.2016.may.05

Gunter, Barrie (2015). News and the Net. London: Routledge. ISBN: 9780805844993

Hall, Jeffrey A. (2018). "When is social media use social interaction? Defining mediated social interaction". New media \& society, v. 20, n. 1, pp. 162-179.

https://doi.org/10.1177/1461444816660782

Hermida, Alfred (2016). Tell everyone: Why we share and why it matters. Toronto, Canada: Anchor Canada. ISBN: ?978 0385679589

Hermida, Alfred; Fletcher, Fred; Korell, Darryl; Logan, Donna (2012). "Share, like, recommend: Decoding the social media news consumer". Journalism studies, v. 13, n. 5-6, pp. 815-824.

https://doi.org/10.1080/1461670X.2012.664430

Jansson, André; Lindell, Johan (2015). "News media consumption in the transmedia age". Journalism studies, v. 16, n. 1, pp. 79-96.

https://doi.org/10.1080/1461670X.2014.890337 
Karapanos, Evangelos; Teixeira, Pedro; Gouveia, Ruben (2016). "Need fulfillment and experiences on social media: A case on Facebook and WhatsApp". Computers in human behavior, v. 55, pp. 888-897.

https://doi.org/10.1016/J.CHB.2015.10.015

Kilgo, Danielle K.; Harlow, Summer; García-Perdomo, Víctor; Salaverría, Ramón (2018). “A new sensation? An international exploration of sensationalism and social media recommendations in online news publications". Journalism, v. 19, n. 11, pp. 1497-1516.

https://doi.org/10.1177/1464884916683549

Kim, Eun-mee; Ihm, Jennifer (2019). "More than virality: Online sharing of controversial news with activated audience". Journalism \& mass communication quarterly, v. 97, n. 1, pp. 118-140.

https://doi.org/10.1177/1077699019836950

Klinger, Ulrike; Svensson, Jacob (2015). "The emergence of network media logic in political communication: A theoretical approach". New media \& society, v. 17, n. 8, pp. 1241-1257.

https://doi.org/10.1177/1461444814522952

Kubey, Robert; Larson, Reed; Csikszentmihalyi, Mihaly (1996). "Experience sampling method applications to communication research questions". Journal of communication, v. 46, n. 2, pp. 99-120.

https://doi.org/10.1111/j.1460-2466.1996.tb01476.x

Kümpel, Anna-Sophie (2019). "The issue takes it all? Incidental news exposure and news engagement on Facebook". Digital journalism, v. 7, n. 2, pp. 165-186.

https://doi.org/10.1080/21670811.2018.1465831

Kümpel, Anna-Sophie; Karnowski, Veronika; Keyling, Till (2015). "News sharing in social media: A review of current research on news sharing users, content, and networks". Social media + society, v. 1, n. 2.

https://doi.org/10.1177/2056305115610141

Kwon, K. Hazel; Moon, Shin-II; Stefanone, Michael A. (2015). "Unspeaking on Facebook? Testing network effects on self-censorship of political expressions in social network sites”. Quality \& quantity, v. 49, n. 4, pp. 1417-1435. https://doi.org/10.1007/s11135-014-0078-8

Lee, Jae-Kook (2009). Incidental exposure to news: Limiting fragmentation in the new media environment. Doctoral dissertation. University of Texas at Austin.

https://repositories.lib.utexas.edu/handle/2152/6686

Lee, Jae-Kook; Kim, Eunyi (2017). "Incidental exposure to news: Predictors in the social media setting and effects on information gain online". Computers in human behavior, v. 75, pp. 1008-1015.

https://doi.org/10.1016/j.chb.2017.02.018

Marwick, Alice E.; Boyd, Danah (2011). "I tweet honestly, I tweet passionately: Twitter users, context collapse, and the imagined audience". New media \& society, v. 13, n. 1, pp. 114-133.

https://doi.org/10.1177/1461444810365313

McNeill, Lynne S. (2018). “'My friend posted it and that's good enough for me!': Source perception in online information sharing". Journal of American folklore, v. 131, n. 522, pp. 493-499.

https://doi.org/10.5406/jamerfolk.131.522.0493

Molyneux, Logan (2018). “Mobile news consumption: A habit of snacking”. Digital journalism, v. 6, n. 5, pp. 634-650. https://doi.org/10.1080/21670811.2017.1334567

Newman, Nic; Fletcher, Richard; Kalogeropoulos, Antonis; Levy, David A. L.; Nielsen, Rasmus-Kleis (2018). Reuters Institute. Digital news report 2018.

http://media.digitalnewsreport.org/wp-content/uploads/2018/06/digital-news-report-2018.pdf

Newman, Nic; Fletcher, Richard; Kalogeropoulos, Antonis; Nielsen, Rasmus-Kleis (2019). Reuters Institute. Digital news report 2019.

https://reutersinstitute.politics.ox.ac.uk/sites/default/files/2019-06/DNR_2019_FINAL_1.pdf

Newman, Nic; Fletcher, Richard; Schulz, Anne; Andi, Simge; Nielsen, Rasmus-Kleis (2020). Reuters Institute. Digital news report 2020.

https://reutersinstitute.politics.ox.ac.uk/sites/default/files/2020-06/DNR_2020_FINAL.pdf

Noguera-Vivo, José-Manuel (2018). "You get what you give: Sharing as a new radical challenge for journalism". Communication \& society, v. 31, n. 4, pp. 147-158.

https://revistas.unav.edu/index.php/communication-and-society/article/view/35678/30180

Papacharissi, Zizi (2015). Affective publics: Sentiment, technology, and politics. Oxford University Press. ISBN: 9780 199999743 
Park, Chang-Sup; Kaye, Barbara K. (2020). "What's this? Incidental exposure to news on social media, News-Finds-Me perception, news efficacy, and news consumption". Mass communication and society, v. 23, n. 2, pp. 157-180. https://doi.org/10.1080/15205436.2019.1702216

Peters, Chris (2015). "Introduction. The places and spaces of news audiences". Journalism studies, v. 16, n. 1. https://doi.org/10.1080/1461670X.2014.889944

Picone, Ike; Courtois, Cédric; Paulussen, Steve (2015). "When news is everywhere. Understanding participation, cross-mediality and mobility in journalism for a radical user perspective". Journalism practice, v. 9, n. 1, pp. 35-49. https://doi.org/10.1080/17512786.2014.928464

Prior, Markus (2007). Post-broadcast democracy: How media choice increases inequality in political involvement and polarizes elections. Cambridge: Cambridge University Press. ISBN: 9781139878425 https://doi.org/10.1017/CBO9781139878425

Rubin, Alan M.; Perse, Elizabeth M. (1987). "Audience activity and television news gratifications". Communication research, v. 14, n. 1, pp. 58-84.

https://doi.org/10.1177/009365087014001004

Salaverría, Ramón; Buslón, Nataly; López-Pan, Fernando; León, Bienvenido; López-Goñi, Ignacio; Erviti, María-Carmen (2020). "Desinformación en tiempos de pandemia: tipología de los bulos sobre la Covid-19". Profesional de la información, v. 29, n. 3, pp. e290315.

https://doi.org/10.3145/epi.2020.may.15

Schrøder, Kim-Christian (2015). “News media old and new: Fluctuating audiences, news repertoires and locations of consumption". Journalism studies, v. 16, pp. 60-78.

https://doi.org/10.1080/1461670X.2014.890332

Segado-Boj, Francisco; Díaz-Campo, Jesús; Navarro-Asencio, Enrique; Remacha-González, Lorena (2020). “Influencia de la percepción 'Las noticias me encuentran' en la evaluación de la exactitud, la factualidad y la relevancia. Caso práctico de noticias sobre el cambio climático”. Revista mediterránea de comunicación, v. 11, n. 2, pp. 85-103.

https://doi.org/10.14198/MEDCOM2020.11.2.12

Shane-Simpson, Christina; Manago, Adriana; Gaggi, Naomi; Gillespie-Lynch, Kristen (2018). "Why do college students prefer Facebook, Twitter, or Instagram? Site affordances, tensions between privacy and self-expression, and implications for social capital". Computers in human behavior, v. 86, pp. 276-288.

https://doi.org/10.1016/j.chb.2018.04.041

Tewksbury, David; Weaver, Andrew J.; Maddex, Brett D. (2001). "Accidentally informed: Incidental news exposure on the world wide web". Journalism and mass communication quarterly, v. 78, n. 3, pp. 533-554.

https://doi.org/10.1177/107769900107800309

Thorson, Kjerstin (2020). "Attracting the news: Algorithms, platforms, and reframing incidental exposure". Journalism, v. 21, n. 8 , pp. $1067-1082$.

https://doi.org/10.1177/1464884920915352

Toff, Benjamin; Nielsen, Rasmus-Kleis (2018). “I just Google it': Folk theories of distributed discovery”. Journal of communication, v. 68, n. 3, pp. 636-657.

https://doi.org/10.1093/joc/jqy009

Trieu, Penny; Bayer, Joseph B.; Ellison, Nicole B.; Schoenebeck, Sarita; Falk, Emily (2019). "Who likes to be reachable? Availability preferences, weak ties, and bridging social capital". Information, communication \& society, v. 22, n. 8, pp. $1096-1111$. https://doi.org/10.1080/1369118X.2017.1405060

Valeriani, Augusto; Vaccari, Cristian (2016). "Accidental exposure to politics on social media as online participation equalizer in Germany, Italy, and the United Kingdom". New media \& society, v. 18, n. 9, pp. 1857-1874.

https://doi.org/10.1177/1461444815616223

Witschge, Tamara; Anderson, Chris W.; Domingo, David; Hermida, Alfred (2018). "Dealing with the mess (we made): Unraveling hybridity, normativity, and complexity in journalism studies". Journalism, v. 20, n. 5, pp. 651-659. https://doi.org/10.1177/1464884918760669

Yang, Chia-Chen (2016). "Instagram use, loneliness, and social comparison orientation: Interact and browse on social media, but don't compare". Cyberpsychology, behavior, and social networking, v. 19, n. 12, pp. 703-708.

https://doi.org/10.1089/cyber.2016.0201

Yuan, Elaine (2011). "News consumption across multiple media platforms. A repertoire approach". Information, communication \& society, v. 14, n. 7, pp. 998-1016.

https://doi.org/10.1080/1369118X.2010.549235 


\section{Anexo}

\section{Texto y preguntas del cuestionario}

\section{Presentación}

En este cuestionario le vamos a pedir que piense en la última noticia o enlace que haya compartido en redes sociales o que haya enviado a una persona, o grupo de personas (por ejemplo, un grupo de WhatsApp, o un grupo de Facebook).

Piense, por favor, en su última publicación de Facebook, el último correo que haya enviado o el último mensaje de mensajería móvil o sms que haya enviado y que contenga un enlace a un contenido o que informe acerca de algún evento.

No tiene por qué tratarse específicamente de una noticia publicada en un medio de comunicación tradicional, puede tratarse de un post de un blog o de cualquier otro contenido

Una vez que tenga identificado ese mensaje que ha enviado, téngalo presente, porque todas las preguntas que le vamos a hacer a continuación estarán referidas a esa noticia o información

\section{BLOQUE 1:}

\section{p1. ¿Leyó la noticia antes de compartirla?}

Respuesta: opción múltiple

Sí, he visitado el enlace al texto completo y he leído la noticia en profundidad

Sí, he visitado el enlace al texto completo, pero he leído la noticia superficialmente, por encima

Solo he leído el titular o la vista previa de la noticia, no he visitado el enlace al texto de la noticia

p2. Por favor, indique la dirección url de la última noticia que haya compartido, sea en Facebook, en mensajería instantánea (WhatsApp, Telegram, Line...), correo electrónico o por cualquier otro medio. Por favor, incluya la url completa, y no solo la web del sitio. Por ejemplo, indique:

https://www.20minutos.es/noticia/3472075/0/china-inaugura-puente-mar-mas-largo-mundo/

en lugar de indicar solo www.20minutos.es.

Si la noticia no enlazaba a ninguna url, por favor, copie el titular o las primeras frases

p3. ¿Cómo ha encontrado esa noticia que ha compartido? Por favor, señale cuál de las siguientes opciones es más correcta Respuesta: opción múltiple

\begin{tabular}{|c|l|}
\hline 1 & Estaba navegando en Internet, sin buscar nada en particular, y me he encontrado con ella \\
\hline 2 & La he encontrado en una web que visito habitualmente para enterarme de las últimas noticias \\
\hline 3 & Estaba buscando información sobre el tema (en Google u otro buscador como Bing) y he encontrado la noticia \\
\hline 4 & Tengo instalada la app del medio de mi teléfono móvil y me ha avisado mediante una notificación automática o personalizada \\
\hline 5 & $\begin{array}{l}\text { Me he enterado por un servicio de alertas del medio al que estoy suscrito, por una newsletter, o por otros medios como el canal de } \\
\text { Telegram del medio }\end{array}$ \\
\hline 6 & $\begin{array}{l}\text { Algún amigo o contacto me ha facilitado el enlace o me ha avisado de la noticia por correo electrónico, mensajería móvil o etiquetán- } \\
\text { dome en redes sociales }\end{array}$ \\
\hline 7 & $\begin{array}{l}\text { Estaba viendo mis redes sociales y he encontrado la noticia, en un mensaje que alguien ha publicado pero que no me mencionaba a } \\
\text { mí directamente, ni en un grupo del que formo parte, }\end{array}$ \\
\hline 8 & Otro (especifique) \\
\hline
\end{tabular}

p4. [Solo para quienes respondieron la opción 6 en la p3] Concretamente, ¿cómo le han hecho llegar esa noticia? Por favor, indique la opción que más se ajuste a la realidad

Respuesta: opción múltiple

\begin{tabular}{|l|}
\hline Por un correo electrónico \\
\hline Por un mensaje privado en una red social (Facebook, Twitter, Instagram...) \\
\hline Alguien me etiquetó en una publicación en Facebook o me mencionó en Twitter para avisarme de la noticia \\
\hline Me mandaron un mensaje privado por una aplicación de mensajería móvil (WhatsApp, Telegram, Line, Facebook Messenger...) \\
\hline Leí la noticia en un grupo privado de mensajería móvil (WhatsApp, Telegram, Line, Facebook Messenger...) \\
\hline Otro (especifique) \\
\hline
\end{tabular}

p5. [Para quienes respondieron la opción 7 en la p3] ¿En qué red social encontró la pregunta?

Respuesta: opción múltiple

- Facebook

- Twitter

- Instagram

- Otro (especifique) 


\section{BLOQUE 2}

p6. ¿Cómo ha compartido esta noticia? Respuesta: opción múltiple

\begin{tabular}{|c|l|}
\hline 1 & En alguna red social (Twitter, Facebook, Instagram...) como una publicación para que la puedan ver todos mis contactos en general. \\
\hline 2 & $\begin{array}{l}\text { En redes sociales, pero me he dirigido a una persona concreta, etiquetándole en Facebook o Instagram o mencionándole en Twitter. } \\
\text { Aun así, el mensaje era público y lo podía ver todos mis contactos }\end{array}$ \\
\hline 3 & $\begin{array}{l}\text { En un grupo privado o comunidad restringida de Facebook o cualquier otra red social, de modo que solo lo puedan ver los miembros } \\
\text { de ese grupo o comunidad }\end{array}$ \\
\hline 4 & En un grupo privado de WhatsApp, Line, Telegram o Facebook Messenger \\
\hline 5 & Se lo he enviado a un contacto concreto mediante mensajería instantánea (WhatsApp, Line, Telegram o Facebook Messenger) \\
\hline 6 & Por correo electrónico, con una persona o grupo de personas determinado \\
\hline 7 & Por correo electrónico, de manera masiva a un gran grupo de personas (más de 50) \\
\hline 8 & Otro (especifique) \\
\hline
\end{tabular}

p7A. [solo para quienes contestaron la opción 1 ó 2 en la p6] Concretamente, ¿̇en qué red social ha compartido esta noticia? (puede indicar más de una, si la ha compartido en más de una)

Respuesta: opción múltiple, se puede marcar más de una opción

- Facebook

- Twitter

- Instagram

- Otro

P7/B [solo para quienes contestaron la opción 4 ó 5 en la p6]. Concretamente, ¿qué aplicación, herramienta o canal ha utilizado para compartir esta noticia? (puede indicar más de una, si la ha compartido en más de una)

- WhatsApp

- Line

- Telegram

- Facebook Messenger

- Otro

P8. A continuación le presentamos una serie de afirmaciones acerca de ese contenido que ha compartido, que nos ha indicado previamente, y del que estamos hablando.

Por favor, indique hasta qué punto está de acuerdo con cada una de estas afirmaciones. 1 significa “Nada de acuerdo” y 5, Totalmente de acuerdo"

1=Nada de acuerdo, 2=Poco de acuerdo, 3=Ni de acuerdo ni de desacuerdo, 4=Bastante de acuerdo, 5=Totalmente de acuerdo

\begin{tabular}{|l|}
\hline Me ha gustado el contenido \\
\hline He disfrutado el contenido \\
\hline El contenido es positivo \\
\hline El contenido es entretenido \\
\hline El contenido es valioso (en general, para mí, para mis amigos o para la sociedad) \\
\hline El contenido es bueno y de calidad \\
\hline El contenido es útil \\
\hline El contenido me ha ayudado a estar informado \\
\hline El contenido es relevante para mi vida \\
\hline
\end{tabular}

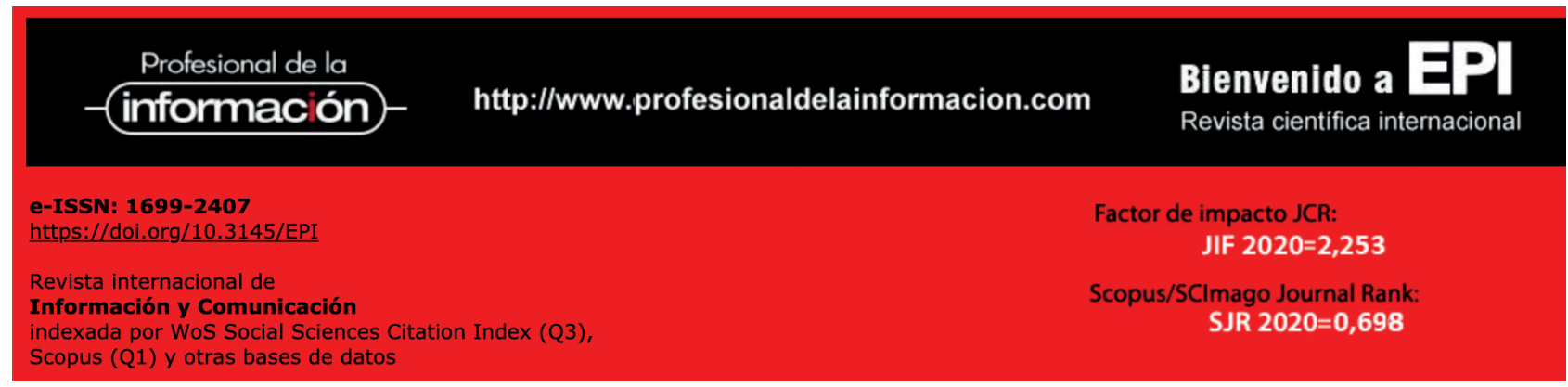

